\title{
Severe sepsis and sepsis shock secondary to non ventilator associated nosocomial pneumonia. principal features and predictors of outcome
}

\author{
R Zaragoza*, N Buceta, S Sancho, C Hurtado, J Camarena, R González, F Puchades, C Martinez, M Cervera \\ From ESICM LIVES 2015 \\ Berlin, Germany. 3-7 October 2015
}

\section{Introduction}

Non ventilator associated nosocomial pneumonia (NVANP) is considered as a rare cause of severe sepsis and septic shock. However its real impact is unknown.

\section{Objectives}

The aims of this study were to describe the principal clinical an epidemiological characteristics of the NVANP severe sepsis and septic shock patients and to describe their clinical and microbiological predictors of outcome.

\section{Methods}

During a year and a half period (January 2013 to July 2014), all NVANP severe sepsis and septic shock consecutively patients in a teaching hospital with sepsis unit were prospectively evaluated, Clinical and microbiological variables were recorded. A univariate analysis was performed to define the factors associated with global mortality (SPSS 20.0). Statistical significance was considered when $p$ value $<0.05$.

\section{Results}

Among 950 severe sepsis and septic shock patients activated, 44 of them corresponded to an episode of NAVNP (4.6\%). Septic shock was present in $31.8 \%$. The principal place of detection was ER in the $77.3 \%$ of the cases. Their mean APACHE II and SOFA score were $19.84 \pm 6.96$ and $5.27 \pm 3.36$ respectively. Microbiological documentation was achieved in $38.6 \%$. Among them the main etiologies were: MRSA (22.2\%); Pseudomonas aeruginosa (22.2\%); MSSA (16.6\%) and S. pneumoniae (16,6\%). $15.9 \%$ of NAVNP were bacteremic and $84.1 \%$ of the episodes received adequate empirical antibiotic treatment (AEAT). Global mortality was $40.9 \%$. In an univariate analysis, presentation as septic shock $(64.2 \%$ vs $40.7 \%, \mathrm{p}=0.03)$, implementation of SSC early resuscitation bundles (3h) $(36.3 \%$ vs $42.4 \%$; $\mathrm{p}=0.05)$ and AEAT $(32.4 \%$ vs $85.7 \%$; $\mathrm{p}=0.02)$ had any impact on prognosis. Neither microbiological documentation ( $47 \%$ vs $37 \%$; $\mathrm{p}=$ 0.30 ) nor bacteremic episodes $(57.1 \%$ vs $36.1 \%, \mathrm{p}=0.27$ ) had any influence on global mortality.

\section{Conclusions}

NAVNP must be considered as a cause of severe sepsis and septic shock and is associated with a poor outcome. Their prognosis is related with the severity of clinical presentation, the implementation of early resuscitation bundles and adequate empirical antibiotic therapy. For these reasons sepsis teams could play a main role in the management of these patiens.

Published: 1 October 2015

doi:10.1186/2197-425X-3-S1-A708

Cite this article as: Zaragoza et al:: Severe sepsis and sepsis shock secondary to non ventilator associated nosocomial pneumonia. principal features and predictors of outcome. Intensive Care Medicine Experimental 2015 3(Suppl 1):A708. 